\title{
Pattern-Stimulated Visual Evoked Potential in Dog: Changes in Elicited Response with Pattern Size and Calculation of Visual Acuity
}

\author{
Yoshiki ITOH $^{1) *}$, Seiya MAEHARA ${ }^{1)}$, Keisuke OKADA ${ }^{1)}$ and Yasuharu IZUMISAWA ${ }^{1)}$ \\ ${ }^{1)}$ Department of Small Animal Clinical Sciences, School of Veterinary Medicine, Rakuno Gakuen University, 582 Bunkyodai-Midorimachi, \\ Ebetsu, Hokkaido 069-8501, Japan
}

(Received 23 February 2010/Accepted 29 June 2010/Published online in J-STAGE 13 July 2010)

ABSTRACT. The aim of this research was to evaluate the changes in the response of pattern-stimulated visual evoked potential (pVEP) with different pattern size, and demonstrate visual acuity from the minimum visual angle. pVEP was recorded from both eyes of six healthy beagles. Prior to $\mathrm{pVEP}$ recording, the dogs were sedated, and a traction fiber was used to prevent the eye from rolling down. The stimulator was set $30 \mathrm{~cm}$ from the subject's eye. Pattern reversal frequency of the stimulating monitor was $3 \mathrm{rev} / \mathrm{sec}$, and pattern size was set at seven levels; 14-364 arc-min (1.2-31.4 mm). Amplitude of the P100 component was evaluated, and visual acuity was calculated from the minimum visual angle to obtain a pVEP response. A pVEP response (2.3-3.1 $\mu \mathrm{V}$ ) was obtained from all subjects. The P100 component was detectable in 3 eyes with a check size of 14 arc-min and 7 eyes with 28 arc-min, and the component was undetectable with 14 arc-min in all subjects in which it was undetectable with 28 arc-min. From the minimum level to obtain the P100 component, the subjects' visual acuity was extrapolated as $0.54-2.14$ cycles per degree. We demonstrated the change in P100 component with check size. However, our technique was inadequate to examine visual acuity because the subject's refractive index was ignored. We suggest that, with further study, pVEP with different check sizes would be applicable for canine visual acuity examination.

KEY WORDS: canine, pattern size, pattern stimulation, visual acuity, visual evoked potential.

J. Vet. Med. Sci. 72(11): 1449-1453, 2010

Visual acuity is the synthetic ability of the eye to resolve the smallest separation between two objects [28]. This ability is one of the most clinically useful single measures of visual function. Visual acuity examination is performed in many clinical situations in human medicine. The most common indicator of visual acuity in human beings is the Snellen fraction, which relates the ability of a subject to discriminate between letters or objects at a fixed distance [26, 28]. This examination in humans requires the subject's response to judge the limit of visual recognition. In veterinary ophthalmology, visual acuity is shown as visual resolution, spatial frequency; the unit is cycles per degree (cpd). However, visual acuity is not used to evaluate an animal's visual sense, because it is impossible to apply subjective visual acuity examination, which is mainly used in humans. The visual sense of animals is evaluated by various responses (e.g., menace and dazzle) and examinations (e.g., maze), and their vision is generally expressed as "visible" or "loss of vision". Though the visual acuity of animals is a curiosity to scientists and ophthalmologists, understanding the degree of an animal's vision is useful in order to know the degree of impairment due to a disorder and the effectiveness of treatment, and for giving detailed clinical information to the animal's owner.

In human medicine, objective visual examination is required to evaluate the pediatric population and neurologically impaired patients, who are impossible to assess subjectively. There are some objective methods for visual acuity

\footnotetext{
* Correspondence to: Itoh, Y., School of Veterinary Medicine,

Rakuno Gakuen University, 582 Bunkyodai-Midorimachi,

Ebetsu, Hokkaido 069-8501, Japan.

e-mail: yoshi66@med.yokohama-cu.ac.jp
}

examination $[19,21,29,30]$, such as using the visual evoked potential (VEP) [29]. VEP is an electrophysiological technique for recording cortical responses by stimulating the retinal cells with a flash or pattern-reversal $[8,9,11]$. This technique plays a role in assessing visual sense, diagnosing visual impairment caused by post-retinal disorders, and evaluating drug-induced disturbance of the visual pathway $[3,5,6,10]$. It is known that pattern-reversal VEP ( $p$ VEP), which uses a checkerboard pattern on a stimulation monitor, is superior to that with flash stimulation in clinical practice, because of the satiability of the values, and amplitude and latency differ with the check size used $[4,11,26$, 29]. The disappearance and/or prolongation of the main component of pVEP, named P100, is utilized to determine visual acuity objectively. There are several reports that Snellen and pVEP acuity show good correlation $[15,16$, 26].

However, there have not been many reports of $\mathrm{pVEP}$ in animals, especially dogs, during the years since the first description of VEP in the dog [13]. Most of the reports of VEP in the dog used flash VEP, because the technique of pVEP would need the subject's cooperation to watch the stimulating monitor. Thus, there is no standard method and data of pVEP in the dog, and, to our knowledge, only one report has described $\mathrm{pVEP}$ for visual acuity examination in the dog [24].

The goal of this research was to establish an objective visual acuity examination for animals, especially the dog. In this study, the change in pVEP with stimulated pattern size was investigated as basic data of pVEP in the dog, and we investigated the possibility of its use for objective visual acuity examination in the dog. 


\section{MATERIALS AND METHODS}

Animals: Twelve eyes of 6 beagle dogs $(3$ males and 3 females; median age, 4.2 years; range, 3 to 5 years; weight, $11.7-15.8 \mathrm{~kg}$ ) were assessed. No abnormalities were recognized on neurologic and ophthalmic examinations before the study, including pupillary light reflex, menace response, tonometry, slit-lamp biomicroscopy, ophthalmoscopy, and electroretinography. This study was conducted according to the guidelines of the experimental animal research committee of Rakuno Gakuen University.

Recording $p V E P$ : Prior to recording, all dogs were sedated with a combination of $0.01 \mathrm{mg} / \mathrm{kg}$ medetomidine (Domitor, Meiji Seika, Tokyo, Japan), $0.15 \mathrm{mg} / \mathrm{kg}$ midazolam (Dormicam, Astellas, Tokyo, Japan), and butorphanol $0.025 \mathrm{mg} / \mathrm{kg}$ (Stadol, Bristol-Myers Squibb, Tokyo, Japan) injected intravenously. Transient pVEP was recorded with a portable VEP system (LE-3000, TOMEY, Aichi, Japan), combined with additional instruments - an amplifier and a recorder. An electroluminescent devised monitor (PS-410, TOMEY, Aichi, Japan) was used as the stimulus display. The details of this monitor were as follows: indicated color: yellow $(580 \mathrm{~nm})$, resolution: $640 \times$ 400 dots, indicated area: $122 \times 195 \mathrm{~mm}$, pixel size: $0.22 \times$ $0.22 \mathrm{~mm}$, frame frequency: $60 \mathrm{~Hz}$, contrast: $75 \%$, mean luminosity: $15 \mathrm{~cd} / \mathrm{m}^{2}$. The stimulus field size subtended a visual angle of $37.25 \times 23.30$ at a testing distance of $30 \mathrm{~cm}$. The subject's eye was supported with a 6-0 silk thread through the dorsal bulbar conjunctiva to gaze at the display. pVEP was recorded after producing mydriasis with a combined solution of $0.5 \%$ tropicamide and $0.5 \%$ phenylephrine hydrochloride (Mydrin-P, Santen, Osaka, Japan). All pupils were fully dilated, to over $13 \mathrm{~mm}, 30 \mathrm{~min}$ after applying mydriatics. During recording, the subject's eye was kept open with a lid retractor, and the contralateral eye was masked by the recorder's hand.

Needle-electrodes were used to record the responses, with the recording electrode positioned at the inion in the midline of the nuchal crest, and the reference at the nasion in the midline of the forehead. A plate-electrode for the ground was attached inside an auricle with conducting paste (EC2 Grass electrode cream, Grass, Rhode Island, United States). The upper limit of impedance was set at less than 5 $\mathrm{ohm}$. The pVEP signal was averaged from 128 repetitions and amplified through high- and low-cut filters with manufacture settings of 0.3 and $100 \mathrm{~Hz}$, respectively.

pVEP was recorded with a stimulation rate of 3 reversals/s $(1.5 \mathrm{~Hz})$. The length of the side of each square pattern was $1.2,2.4,4.9,7.3,12.2,19.5$ and $31.7 \mathrm{~mm}$, and the visual angle was 14, 28, 56, 84, 140, 224 and 364 arc minutes (arc-min), respectively. Recording always started with the smallest check size of 14 arc-min and used the largest size in each eye of each dog in a dimly light room.

pVEP evaluation and statistical analysis: $\mathrm{P} 100$ of the fVEP component was measured according to the standard report of the International Society for Clinical Electrophysiology of Vision [23]. We computed the amplitude of P100.
pVEP was recorded twice for each check size, and mean values were calculated. The amplitude of P100 was statistically analyzed with Spearman's correlation coefficient by rank, using GraphPad Prism 5 software (GraphPad Software Inc., California, United States) for Windows. The statistical significance of differences was determined with a $p$-value ( $p$ ) less than 0.05 as the minimum acceptable level of significance.

Extrapolation of visual acuity: The minimum visual angle to obtain the P100 component was used as an indicator to extrapolate visual acuity of the subjects' eyes. Visual acuity was extrapolated from the visual angle according to following formula, derived from Borish [2]:

$$
\text { Cycles per degree }(\mathrm{cpd})=30.0 / \text { arc-min }
$$

\section{RESULTS}

In all animals, sedation and thread placement through the conjunctiva were adequate to perform pVEP recording. Characteristic $p$ VEP waveforms with each check size are shown in Fig. 1. pVEP was recorded from all subjects stimulated with a check size of 56 arc-min or greater. With a smaller check size, the P100 component was detectable in 3 eyes with a check size of 14 arc-min and 7 eyes with 28 arcmin, and the component was undetectable with 14 arc-min in all subjects in which it could not be detected with 28 arc$\min$.

The results are shown in Table 1, as the mean of 12 eyes for each check size. The amplitude was $2.3-3.1 \mu \mathrm{V}$. Highest amplitudes were obtained with medium sizes of stimulus check, 56-140 arc-min. In spite of the larger check size, the amplitude of P100 with 224 and 364 min-arc was decreased. No significant correlation between amplitude and check size was observed. No significant correlation was seen between amplitude and check size.

The putative visual acuity of each subject was computed and is shown in Table 2; in 3 eyes the minimum level to obtain P100 with 14 arc-min was 2.14 cpd; in 4 eyes with 28 arc-min it was $1.07 \mathrm{cpd}$; and in 5 eyes with 56 arc-min it was $0.54 \mathrm{cpd}$.

\section{DISCUSSION}

Subjective examination of visual acuity is impossible in animals, especially in our clients' dogs and cats; thus, evaluation must be performed objectively. In the present study, we performed $\mathrm{pVEP}$ recording with different sizes of pattern stimulus to investigate and establish objective examination of visual acuity in the dog. A pVEP response was obtained from all subjects, and the P100 component disappeared with smaller pattern size, especially 14 and 28 arcmin, in some subjects. We could calculate the visual acuity of each eye in each subject from the minimum level to obtain the P100 component. Our subjects' visual acuity with this method was extrapolated as $0.54-2.14 \mathrm{cpd}$.

Visual acuity in humans is the most important issue in 
(arc-min)

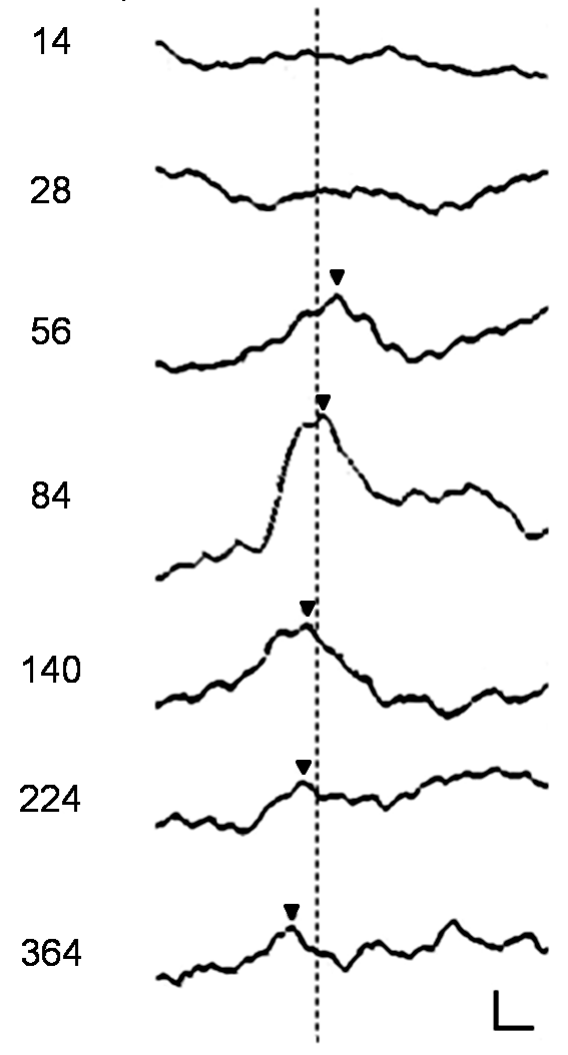

Fig. 1. Characteristic pVEP waveforms with each check size. Calibration: horizontal $25 \mathrm{~ms}$, vertical $2 \mu \mathrm{V}$. Dotted line means $100 \mathrm{~ms}$. Triangular marks indicate P100 component of pVEP. The P100 component elicited with check sizes of 14 and 28 arc-min was undetectable in 9 eyes and 5 eyes, respectively. An "undetectable" pattern with 14 and 28 arc-min is shown here. Stimulating check size of 56,84 and 140 arc-min was associated with a comparatively higher amplitude of P100.

clinical ophthalmology, and reflects the patient's condition. Examination of visual acuity is frequently performed in human medicine. In veterinary ophthalmology, however, although it logically has the same importance, it has been largely ignored, especially in clinical situations. Visual function is usually evaluated by the neural reflex and/or behavior [27]. If the visual acuity of animals were assessed as in human medicine, this parameter would be useful in clinical and experimental situations.

Neurons of the visual cortex in the cerebrum have higher sensitivity to visual simulation with an outline of a figure and contrast than with uniform flash stimulation of the retina [1]. Pattern reversal stimulation, with alternate switching of grid color, affects neurons of the visual cortex, even with weak light energy $[8,11]$. In this research, the P100 component was seen at around $100 \mathrm{~ms}$ by stimulating with a check size of 56 arc-min or greater. On stimulating with 14
Table 1. Amplitude and latency of P100 with each check size

\begin{tabular}{ccc}
\hline $\begin{array}{c}\text { Check size } \\
(\text { arc-min })\end{array}$ & $\begin{array}{c}\text { Amplitude } \\
(\mu \mathrm{V})\end{array}$ & \begin{tabular}{c}
$(n=12$ eyes $)$ \\
\hline 14
\end{tabular} \\
\hline $2.3 \pm 0.7$ & $3 *(25.0 \%)$ \\
56 & $2.9 \pm 0.9$ & $7(58.3 \%)$ \\
84 & $3.1 \pm 1.3$ & $12(100 \%)$ \\
140 & $3.1 \pm 0.9$ & $12(100 \%)$ \\
224 & $3.1 \pm 1.0$ & $12(100 \%)$ \\
364 & $2.6 \pm 0.6$ & $12(100 \%)$ \\
\hline
\end{tabular}

Values are mean \pm SD. The P100 component was undetectable in 9 eyes with a check size of 14 arc-min, and 5 eyes with 28 arc-min. Hence, the values of the two points are mean \pm SD of 3 and 7 eyes, respectively.

*: The 3 eyes were included in the 7 eyes that detected the P100 component with a check size of 28 arc-min.

Table 2. Putative visual acuity derived from $\mathrm{pVEP}$ in dogs

\begin{tabular}{cccc} 
& & & $(n=12$ eyes $)$ \\
\hline Arc-min * & 14 & 28 & 56 \\
\hline $\begin{array}{c}\text { Putative visual acuity (cpd) } \\
\text { Number of eyes }\end{array}$ & 32.14 & 1.07 & 0.54 \\
\hline
\end{tabular}

*: Minimum visual angle to obtain P100 component.

' Putative visual acuity was calculated by the formula:

Cycles per degree $(\mathrm{cpd})=30.0 /$ arc-min

and 28 arc-min, the $\mathrm{P} 100$ component disappeared. It is reported that the $\mathrm{P} 100$ component is around $100 \mathrm{~ms}$ in humans when the subject recognizes the stimulus pattern well [22]. We consider that our subjects found it difficult to recognize the two pattern sizes, as in humans. P100 amplitude showed wide variation with each pattern size because of individual differences. It is unknown why in our results the amplitude was not higher with a stimulus-pattern size of 56 arc-min or greater. In reporting of canine pVEP, P100 amplitude is difficult to evaluate because of instability [24]. The factors influencing $p$ VEP in the dog are unclear because of the small number of reports. We recorded pVEP under sedation with a yellow-black pattern in this research; however, it was difficult to maintain a constant level of consciousness. There are no reports of canine pVEP using a yellow-black check pattern. Odom et al. used a white-black check pattern in previous reports [24]. In human studies, it was reported that $\mathrm{pVEP}$ could be recorded normally with a yellow-black pattern, as with a white-black pattern [18], and drowsiness causes less recognition and affects pVEP [12, $22,31]$. Thus, we consider that sedation and check pattern color were two factors causing dispersion of amplitude in the present study.

Evaluation of visual acuity in the dog using behavioral methods [20], optokinetic nystagmus [7], pattern electroretinography $[24,25]$ and pVEP has been reported [24]. These techniques apply the response of the retina or visual cortex to external light, as in our method. Each method has its own units for expressing visual acuity, and those units are com- 
parable, following Borish's report [2]. Estimates of canine visual acuity varied widely in these reports, 4.3-11.6 cpd. This could be due to different conditions for examination (i.e., stimulating light intensity, monitor contrast, correction of refractive index, breed, state of sedation, or consciousness). In our results, the P100 component was undetected with small check sizes, 14 and 28 arc-min, in some subjects. We consider that a check size of 14-56 arc-min was the limit for visual recognition in our subjects, because the P100 component disappeared with 14 and 28 arc-min. This phenomenon has been reported in humans, because of poor recognition of the pattern stimulus [12, 22, 31]. In addition, the P100 component was undetectable with 14 arc-min in all subjects in which it was undetectable with 28 arc-min. This result provides proof that our method reflected the subjects' visual recognition. The 3 eyes in which P100 was detected with 14 arc-min would have had superior visual acuity. From these results, our subjects' visual acuity was equivalent to $0.54-2.14 \mathrm{cpd}$, from the minimum visual angle. However, these values were much lower than those previous reported [7, 20, 24, 25]. Thus, we consider that the visual acuity obtained from our results does not express true visual resolution.

It is reported that refractive error and stimulus patternsize are large factors in pVEP and visual acuity [14, 22]. Clear vision is results from the image on the retina. During pVEP recording, the refractive index of the subject's eye was stable because of accommodative palsy induced by a mydriatic. However, accommodative palsy may cause poor recognition of the stimulus. In addition, the refractive error shows variation among species, breeds, and individuals [17]. Interestingly, there are no reports on the refractive error of beagles. Refractometry is not a common technique in veterinary ophthalmology; however, this factor should be investigated to establish objective examination of true visual acuity with pVEP in the dog. Correction of the subject's refractive error would be possible using a contact lens. In a further study, correction of the refractive index and an adequate distance for the image to focus on the subject's retina are needed for extrapolation of visual acuity in the dog.

In conclusion, we demonstrated the VEP response with various pattern sizes in the dog, and disappearance of the P100 component by stimulation with smaller check size. Objective examination of visual acuity with pVEP in humans applies this characteristic change in the pVEP component. However, our technique was not sufficient for examination of canine visual acuity because the subject's refractive error was ignored. Thus, our results showed basic data of pVEP in the dog. We suggest that, with further study, $\mathrm{pVEP}$ recording with different check sizes would be applicable for canine visual acuity examination.

ACKNOWLEGMENT. This study was supported by a Grant-in-Aid for Scientific Research from the Japanese Society for the Promotion of Science (No. 21780292).

\section{REFERENCES}

1. Ariwli, A., Sterkin, A., Grinvald, A. and Aertsen, A. 1996. Dynamics of ongoing activity: Explanation of the large variability in evoked cortical responses. Science 273: 1868-1871.

2. Borish, I. M. 1975. Visual acuity. pp. 370-378. In: Clinical refraction, Vol. 1. 3rd ed. (Borish I. M. ed.), Professional Press Inc, Chicago.

3. Cartner, S. C., Barlow, S. C. and Ness, T. J. 2007. Loss of cortical function in mice after decapitation, cervical dislocation, potassium chloride injection, and $\mathrm{CO}_{2}$ inhalation. Comp. Med. 57: $570-573$.

4. Cobb, W. A., Morton, H. B. and Ettinger, G. 1967. Cerebral potentials evoked by pattern reversal and vein suppression in visual rivalry. Nature 216: 1123-1125.

5. Derin, N., Akpinar, D., Yargicoglu, P., Agar, A. and Aslan, M. 2009. Effect of alpha-lipoic acid on visual evoked potentials in rats exposed to sulfite. Neurotoxicol. Teratol. 31: 34-39.

6. Di Loreto, V., Rigalli, A., Cinara, L. and Hernández, G. 2008. Effect of disodium monofluorphosphate on plasma and blood viscosity in the rat. Clin. Hemorheol. Microcirc. 40: 259-265.

7. Ezeh, P. I., Myeres, L. J., Cummins, K. A. and Whitley, R. D. 1990. Utilizing an optokinetic device in assessing the functional visual acuity of the dog. Prog. Vet. Neurol. 1: 427-432.

8. Fox, M. W. 1968. Neuronal development and ontogeny of evoked potentials in auditory and visual cortex of the dog. Electroencephalogr. Clin. Neurophysiol. 24: 213-226.

9. Haliday, A. M. and Mushin, J. 1980. The visual evoked potential in neuroophthalmology. Int. Ophthalmol. Clin. 20: 155183.

10. Hara, H., Ichikawa, M., Oku, H., Shimazawa, M. and Araie, M. 2005. Bunazosin, a selective alpha1-adrenoceptor antagonist, as an anti-glaucoma drug: effects on ocular circulation and retinal neuronal damage. Cardiovasc. Drug Rev. 23:43-56.

11. Harding, G. F. A. 1974. The visual evoked response. Adv. Ophthalmol. 28: 2-28.

12. Harter, M. R. and White, C. T. 1968. Effects of contour sharpness and check size on visually evoked cortical potentials. Vision Res. 8: 701-711.

13. Howard, D. R. and Breazile, J. E. 1972. Normal visual cortical evoked response in the dog. Am. J. Vet. Res. 33: 2155-2157.

14. Katsumi, O., Arai, M., Wajima, R., Denno, S. and Hirose, T. 1996. Spatial frequency sweep pattern reversal VER acuity vs Snellen visual acuity: Effect of optical defocus. Vision Res. 36: 903-909.

15. Katsumi, O., Mehta, M. C., Larson-Park, E. W., Skladzien, C. J. and Hirose, T. 1994. Pattern reversal visual evoked response and Snellen visual acuity. Graefes Arch. Clin. Exp. Ophthalmol. 232: 272-278.

16. Keyser, M. D., Vissenberg, I. and Neetens, A. 1990. Are visually evoked potentials (VEP) useful for determination of visual acuity? - A clinical trial. Neuroophthalmology 10: 153-163.

17. Kubai, M. A., Bentley, E., Miller, P. E., Mutti, D. O. and Murphy, C. J. 2008. Refractive states of eyes and association between ametropia and breed in dogs. Am. J. Vet. Res. 69: 946951.

18. Li, Z., Kita, K., Takahashi, H. and Oguchi, Y. 1993. Clinical application of portable VEP and ERG equipment PE-400. Ganka 35: 281-286 (in Japanese).

19. Mohindra, I., Zwaan, J., Held, R., Brill, S. and Zwaan, F. 1985. Development of acuity and stereopsis in infants with esotropia. Ophthalmology 92: 691-697.

20. Neuhaus, W. and Regenfuss, E. 1967. Über die sehscharfe jdes 
haushundes bei verschiedenen helligkeiten. Zntralbl. Vergleich. Physiol. 57: 137-146.

21. Norica, A. and Tyler, C. 1985. Spatial frequency sweep VEP. Visual acuity during the first year of life. Vision Res. 25: 13991408.

22. Odom, J. V., Bach, M., Brigell, M., Holder, G. E., McCulloch, D. L., Tormene, A. P. and Vaegan. 2003. Visual evoked potentials standard. pp. 301-308. In: Principles and Practice of Clinical Electrophysiology of Vision, 2nd ed. (John, R. H. and Geoffrey, B. A. eds.), MIT PRESS, London.

23. Odom, J. V., Bach, M., Brigell, M., Holder, G. E., McCulloch, D. L., Tormene, A. P. and Vaegan. 2010. ISCEV standard for clinical visual evoked potentials (2009 update). Doc. Ophthalmol. 120: 111-119.

24. Odom, J. V., Bromberg, N. M. and Dawson, W. W. 1983. Canine visual acuity: retinal and cortical field potentials evoked by pattern stimulation. Am. J. Physiol. 245: R637-641.

25. Ofri, R., Dawson, W. W. and Gelatt, K. N. 1993. Visual resolution in normal and glaucomatous dogs determined by pattern electroretinogram. Prog. Vet. Comp. Ophthalmol. 3: 111-116.

26. Ohn, Y., Katsumi, O., Matsui, Y., Tetsuka, H. and Hirose, T.
1994. Snellen visual acuity versus pattern reversal visualevoked response acuity in clinical applications. Ophthalmic. Res. 26: 240-252.

27. Ollivier, F. J., Plummer, C. E. and Barrie, K. P. 2007. Ophthalmic Examination and diagnosis: The eye examination and diagnostic procedures. pp. 438-483. In: Veterinary ophthalmology 4th ed. (Gelatt, K. N. ed.), Blackwell Publishing, Ames.

28. Pirenne, M. H. 1962. Visual acuity. pp. 175-195. In: The Eye. (Davson. H. ed.), Academic, New York.

29. Sokol, S. and Dobson, V. 1976. Pattern reversal visually evoked potentials in infants. Invest. Ophthalmol. Vis. Sci. 15: $58-62$.

30. Wester, S. T., Rizzo, J. F. III, Balkwill, M. D. and Wall, C. III. 2007. Optokinetic nystagmus as a measure of visual function in severely visually impaired patients. Invest. Ophthalmol. Vis. Sci. 48:4542-4548.

31. Yamazaki, H. and Adachi-Usami, E. 1988. Aging effects on spatial frequency Characteristics measured by VECPs. Acta Soc. Ophthamol. Jpn. 92: 1662-1665. 